\title{
Does diabetes really cause bone disease?
}

\author{
Friedrich C. Luft
}

Published online: 3 October 2012

(C) Springer-Verlag Berlin Heidelberg 2012

There are no two more complex chronic conditions than diabetes and osteoporosis. Diabetes is a generic term and is commonly divided into type 1 , an autoimmune, insulindeficiency disease, and type 2, which is hallmarked by insulin resistance and initially at least by high insulin levels. The latter condition may afflict half a billion persons worldwide and is associated with relative obesity. All diabetics are at risk of developing peripheral neuropathy so that fracture risk from falls and neuropathic bone disease is substantially increased. Thus, there are numerous confounders in any relationship between diabetes and bone disease.

In this issue, Freude et al. [1] report that hyperinsulinemia reduces osteoblast activity in vitro via upregulation of transforming growth factor-beta (TGF- $\beta$ ). They incubated human osteoblasts under 8 - and $25-\mathrm{mM}$ glucose conditions, with or without insulin. Insulin increased cell proliferation and decreased alkaline phosphatase activity under both glycemic conditions. The authors then found that osteogenic matrix mineralization of osteoblasts was not influenced by degrees of glycemia but was reduced by insulin; the reduction corresponded to the effects on alkaline phosphatase. Furthermore, TGF- $\beta$ secretion was increased by insulin stimulation. The authors next observed that by blocking TGF- $\beta$, they could restore alkaline phosphatase activity. Subsequently, they compared gene expression in their cells and observed that similar gene expression-related effects were engendered by insulin and TGF- $\beta$. They then performed western blotting and observed that insulin and glucose treatment decreased the runt-related transcription factor-2 (RUNX2), osteoprotegerin, osteocalcin, and

F. C. Luft $(\bowtie)$

Charité Medical Faculty and the Max-Delbrück Center for

Molecular Medicine, Experimental and Clinical Research Center,

Lindenbergerweg 80,

13125 Berlin, Germany

e-mail: luft@charite.de osteonectin expressions. Inhibiting the activin receptor-like kinase (Alk5) abrogated these effects. Alk5 has a Smadactivating function and is necessary for TGF- $\beta$ signaling. The authors suggest that their human osteoblasts exhibited a loss of function engendered by continuous high insulin levels. This functional loss featured increased TGF- $\beta$ signaling that could favor osteoclastogenesis. As a result, impaired bone formation could result.

The cell culture results appear impressive. However, what is the evidence that diabetes per se causes osteopathy? In support of their argument, the authors cite reviews on the topic $[2,3]$. Hip fracture risk is indeed increased in type 1 and type 2 diabetes; however, bone mineral density (BMD) is decreased in type 1 diabetes but actually increased in type 2 diabetes [3]. Tuominen and colleagues [4] measured BMD in diabetic patients with dual-energy x-ray absorptiometry. They found that between both sexes, BMD values were significantly lower in type 1 diabetic patients than in type 2 diabetic patients or the control subjects. When adjusted for age and body mass index, the differences between type 1 diabetic patients and control subjects remained essentially unchanged in both sexes, whereas the differences between type 1 and type 2 diabetic subjects were significant only in men. After further adjustments for confounding factors, the average BMD values were still lower in type 1 diabetic subjects than in type 2 diabetic subjects although with lesser significance. Past low-energy fractures were more common in type 1 diabetic women than in type 2 diabetic women. The authors concluded that the lower BMD in type 1 versus type 2 diabetic patients and control subjects probably resulted from more rapid bone loss after the onset of type 1 diabetes mellitus. They could not explain this state of affairs by insulin treatment, which was prescribed for both types of patients in their study. Because the causes of low BMD in type 1 diabetes are unknown, the authors suggested that these patients should be evaluated for the risk of 
osteoporosis and related fractures and offered appropriate preventive measures. They made no such recommendations for patients with type 2 diabetes that would conceivably better fit into the cell culture results.

Any relationship between osteoporosis and type 2 diabetes mellitus remains murky [5]. Another meta-analysis of this issue involving 47 studies found that 26 studies actually showed increased BMD in type 2 diabetic patients. Another 13 studies reported decreased BMD, and 8 studies found no difference in BMD comparing patients to controls. An alternative approach to bone-related diseases and type 2 diabetes mellitus could be through a genetic analysis. Billings et al. [6] explored genetic pleiotropy as a possible mechanistic association. They analyzed 19,000 individuals and assessed gene expression. They queried single nucleotide polymorphisms (SNPs) associated with BMD at levels of genome-wide significance, variants in linkage disequilibrium, and BMD candidate genes. They found that SNP rs6867040, at the integrin alpha 1 subunit (ITGA1) locus, was associated with a $0.0166-\mathrm{mmol} / \mathrm{L}$ increase in fasting glucose per $\mathrm{C}$ allele in their combined analysis. Furthermore, genetic variants in the ITGA1 locus were associated with its expression in the liver but not in white adipose tissue. ITGA1 variants appeared among the top loci associated with type 2 diabetes, fasting insulin, $\beta$ cell function by homeostasis model assessment, and 2-h post-oral glucose tolerance test glucose and insulin levels. Since ITGA1 has demonstrated genetic pleiotropy in prior studies, and because of its suggested role in liver fibrosis, insulin secretion, and bone healing, the authors suggest that ITGA1 lends credence to its contribution to both osteoporosis and type 2 diabetes. ITGA1 develops heterodimers with the beta-1 subunit to form a cell-surface receptor for collagen and laminin. The heterodimeric receptor is involved in cell-cell adhesion and may play a role in inflammation and fibrosis. The alpha 1 subunit contains an inserted (I) von Willebrand factor type I domain, which may be involved in collagen binding.

An alternative approach to the problem could be the use of an animal model. The Zucker (fatty) rat is one of a group of animals that inherits obesity as an autosomal Mendelian recessive trait. These rats are obese, hyperphagic, and hyperinsulinemic. Rosiglitazone (RSG) is an insulin-sensitizing drug used to treat type 2 diabetes mellitus. The "A Diabetes Outcome Progression Trial" (ADOPT) showed that type 2 diabetic women taking RSG experienced more fractures than patients taking other type 2 diabetes drugs. These were not osteoporotic vertebral fractures but instead occurred in the limbs. Sardone et al. [7] recently used the Zucker rat model to explore the relationship. The authors found that RSG decreased bone quality. An unusual finding in their study was an increase in cortical bone porosity induced by RSG, consistent with its effect on long bones of women.
Alendronate, an inhibitor of bone reabsorption, enhanced mechanical strength in the model and may provide an approach to partially counter the deleterious skeletal effects of RSG. The Zucker rat is leptin deficient. This fact has specific ramifications since osteoporosis is less common in individuals with high fat mass. Zaidi et al. recently drew attention to the fact that adipokines, such as leptin, modulate bone cells through both direct and indirect actions, whereas molecules activating peroxisome proliferator-activated receptor $\gamma$ drive mesenchymal stem cell differentiation toward adipocytes away from the osteoblastic lineage [8]. They suggest that bone-derived osteocalcin regulates insulin release and insulin sensitivity and hence might indirectly affect fat mass.

Tamasi et al. [9] characterized the bone structure in the Zucker rat. They used micro computerized tomography (microCT) and found that trabecular architecture measured by microCT indicates a trend toward decreasing bone volume in the obese animals, evident by a decrease in trabecular number and thickness with an increase in trabecular separation. Histomorphometric evaluation further demonstrated increases in the osteoclast surface in the obese rats at both 9 and 15 weeks without a change in osteoclast number. Nonetheless, the changes were subtle. The technique is superb, and an example of a human so studied is provided to exemplify the technique (Fig. 1).

In models of type 1 diabetes mellitus, the relationship between diabetes and bone disease appears a bit more straightforward. The nonobese diabetic (NOD) mouse model develops diabetes spontaneously. Unfortunately, in this model, the onset of diabetes is unpredictable, occurs in a minority of male mice, and can only be studied in a single mouse strain. To validate the relevance of the more flexible

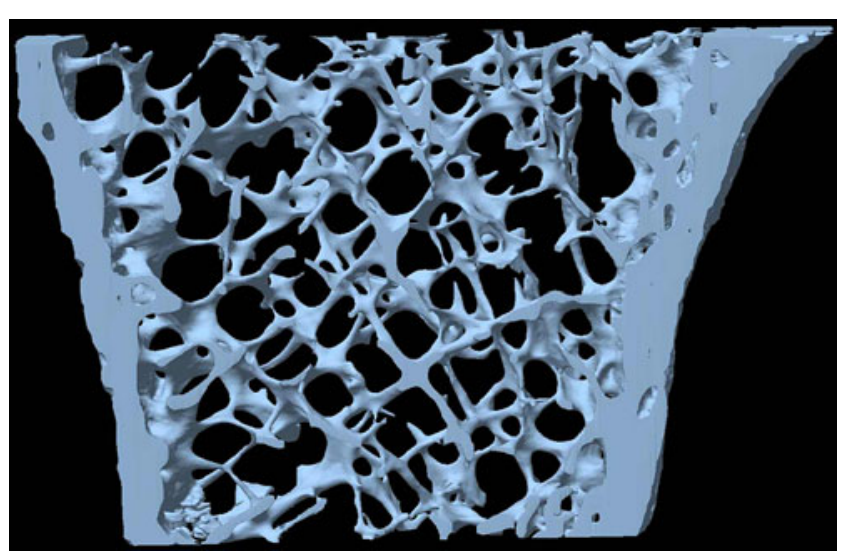

Fig. 1 Microcomputed tomography of bone can produce pictures such as this giving great detail on morphology of osteoporosis. This section is from a trans-iliac bone biopsy from a patient with osteoporosis. Similar studies have been done in rat models of diabetes mellitus [9]. With courtesy from GEROM - Angers (Prof. D. CHAPPARD), see web page: http://med2.univ-angers.fr/discipline/lab histo/page microCT.htm 
streptozotocin-induced diabetes model for studying diabetes-associated bone loss, Botolin and McCabe compared its phenotype to the spontaneously diabetic NOD model [10]. Both models exhibited hyperglycemia and loss of body, fat pad, and muscle weight. Furthermore, these genetically different and distinct models of diabetes induction demonstrated similar bone phenotypes marked by significant trabecular bone loss and increased bone marrow adiposity. Both diabetic models also exhibited decreased osteocalcin mRNA and increased adipocyte fatty acidbinding protein 2 mRNA levels in isolated tibias and calvaria. The authors concluded that multiple streptozotocin injection-induced diabetes is a valid model for understanding the acute and chronic pathophysiologic responses to diabetes and their mechanisms in bone. Their observations in two type 1 diabetes mellitus models support a relationship between this kind of diabetes and bone disease, but has no relevance to any actions of insulin on osteoblasts since insulin was highly deficient in these models.

What can we conclude from these diverse findings? We must accept the cell culture results of Freude et al. [1] at face value. How this information can best be translated into relevance for patients will require much more study, particularly in various animal models.

Respectfully, Friedrich C. Luft

\section{References}

1. Freude F, Braun KF, Haug A, Pscherer S, Stöckle U, Nussler AK, Ehnert S (2012) Hyperinsulinemia reduces osteoblast activity in vitro via upregulation of TGF- $\beta$. J Mol Med. doi:10.1007/s00109012-0948-2

2. Retzepi M, Donos R (2010) The effect of diabetes mellitus on osseous healing. Clin Oral Implants Res 21:673-681

3. Vestergaard P (2007) Discrepancies in bone mineral density and fracture risk in patients with type 1 and type 2 diabetes - a metaanalysis. Osteoporos Int 18:427-444

4. Tuominen JT, Puukka P, Impivaara O, Ronnenmaa T (1999) Bone mineral density in patients with type 1 and type 2 diabetes. Diabetes Care 22:1196-1200

5. Abdulameer SA, Sulaiman SA, Hassali MA, Subramaniam K, Sahib MN (2012) Osteoporosis and type 2 diabetes mellitus: what do we know and what we can do? Patient Prefer Adherence 6:435-448

6. Billings LK, Hsu YH, Ackerman RJ, Dupuis J, Voight BF, RasmussenTorvik LJ, Hercberg S, Lathrop M, Barnes D, Langenberg C et al (2012) Impact of common variation in bone-related genes on type 2 diabetes and related traits. Diabetes 61(8):2176-2186

7. Sardone LD, Renlund R, Willett TL, Fantus IG, Grynpas MD (2011) Effect of rosiglitazone on bone quality in a rat model of insulin resistance and osteoporosis. Diabetes 60(12):3271-3278

8. Zaidi M, Buettner C, Sun L, Iqbal J (2012) Minireview: the link between fat and bone: does mass beget mass? Endocrinology 153:2070-2075

9. Tamasi JA, Arey BJ, Bertolini DR, Feyen JH (2003) Characterization of bone structure in leptin receptor-deficient Zucker (fa/fa) rats. J Bone Miner Res 18:1605-1611

10. Botolin S, McCabe LR (2007) Bone loss and increased bone adiposity in spontaneous and pharmacologically induced diabetic mice. Endocrinology 148:198-205 